\title{
A randomized controlled study of anterior lumbar plexus combined with parasacral sciatic nerve block under the guidance of ultrasound and nerve stimulator in elderly patients undergoing hip arthroplasty
}

\section{Wei Wu}

CR \& WISCO General Hospital, Affiliated to Wuhan University of Science and Technology

Wen-hao Bu ( $\square$ buwenhao2011@163.com )

Maternal and Child Health Hospital of Hubei Province,Tongji Medical College, Huazhong University of

Science and Technology

Hai-bin Huang

CR \& WISCO General Hospital, Affiliated to Wuhan University of Science and Technology

\section{Ying Mao}

CR \& WISCO General Hospital, Affiliated to Wuhan University of Science and Technology

\section{Yong-xing Tan}

Affiliated Hospital of Guilin Medical University

\section{Research Article}

Keywords: ultrasound-guided, anterior lumbar plexus, parasacral sciatic nerve block, hip arthroplasty, elderly

Posted Date: April 5th, 2021

DOI: https://doi.org/10.21203/rs.3.rs-308699/v2

License: (c) (1) This work is licensed under a Creative Commons Attribution 4.0 International License.

Read Full License 


\title{
A randomized controlled study of anterior lumbar plexus combined with parasacral sciatic nerve block under the guidance of ultrasound and nerve stimulator in elderly patients undergoing hip arthroplasty
}

\author{
Wei $\mathrm{Wu}^{1,3}$,Wen-hao $\mathrm{Bu}^{2}$,Hai-bin Huang ${ }^{1}$, Ying $\mathrm{Mao}^{1}$,Yong-xing Tan ${ }^{3,4}$
}

1. Department of Anesthesiology, CR \& WISCO General Hospital, Affiliated to Wuhan University of Science and Technology; No.209 Yejin Road,Wuhan, 430080,China

2. Department of Anesthesiology, Maternal and Child Health Hospital of Hubei Province, Tongji Medical College, Huazhong University of Science and Technology; No. 745 Wuluo Road, Wuhan, 430070,China

3. Guilin Medical University;No.1 Zhiyuan Road,Guilin,541000,China

4. Affiliated Hospital of Guilin Medical University; No.15 Lequn Road,Guilin,541000,China

First author: Wei WuＥ-mail: wuwei201106@163.com

Corresponding author: Wen-hao Bu E-mail: buwenhao2011@163.com

\begin{abstract}
Objectives:To explore the clinical application of anterior lumbar plexus combined with parasacral nerve block under the dual guidance of ultrasound and nerve stimulator in elderly hip arthroplasty.

Methods:Methods:Forty elderly patients aged from 65 to 85 years old who underwent unilateral hip arthroplasty (Except for revision of hip joint) were randomly divided into two groups ( $\mathrm{n}=20$ in each group): ultrasound-guided anterior lumbar plexus combined with parasacral sciatic nerve block group (group A) and ultrasound-guided anterior lumbar plexus block group (group B). Both groups were assisted with sedation with different doses of dexmetopyrimidine and analgesia with different doses of sufentanil. Because the orthopaedic team of our hospital performed hip arthroplasty with mature technique and fixed operation time, the onset time of block in group An and $\mathrm{B}$, heart rate and blood pressure at admission (T0), skin incision (T1), half hour after skin incision (T2) and skin suture(T3), intraoperative dosage ofdexmedetomidine and sufentanil, postoperative $1,4,8,16,24 \mathrm{hVAS}$ score and the first time of getting out of bed were recorded.
\end{abstract}

Results: the heart rate and blood pressure at T1, T2 and T3 in group A were significantly lower than those in group $\mathrm{B}(\mathrm{P}<0.05)$. The amount of sufentanil used during operation in group), A was significantly lower than that in group $\mathrm{B}(\mathrm{P}<0.05)$. The postoperative scores of $1,4,8,16$ and $24 \mathrm{hVAS}$ in group), A were significantly lower than those in group B $(\mathrm{P}<0.05)$, and the time of getting out of bed for the first time after operation was shorter than that in group $\mathrm{B}(\mathrm{P}<0.05)$.

Conclusion: compared with simple anterior lumbar plexus block, anterior lumbar plexus combined with parasacral sciatic nerve block in elderly patients with hip arthroplasty can make the intraoperative circulation more stable, the intraoperative dosage of opioids significantly reduced and the effect of early postoperative analgesia 
is better. it can also shorten the time for patients to get out of bed for the first time.

Key words: ultrasound-guided; anterior lumbar plexus; parasacral sciatic nerve block; hip arthroplasty; elderly

\section{Introduction}

Most of the elderly suffer from osteoporosis, coupled with the degeneration of the muscles around the hip joint, slow response, can not effectively offset the nociceptive stress of the hip, so the risk of femoral neck fracture is higher [1]. Hip arthroplasty is a common method for the treatment of femoral neck fracture. Hip replacement patients are generally older, often accompanied by diabetes, hypertension, heart disease and respiratory diseases, so the risk of anesthesia is higher. Ultrasound-guided peripheral nerve block can increase the success rate of anesthesia, reduce related complications, and reduce the effect of routine anesthesia on respiratory and circulatory function of patients. It is often used in elderly patients and patients with poor cardiopulmonary function $[2,3]$. The purpose of this study was to observe the anesthetic effect of ultrasound-guided anterior lumbar plexus combined with parasacral sciatic nerve block in elderly patients undergoing hip arthroplasty.

\section{Data and Methods}

\subsection{Data}

A total of 458 cases of hip arthroplasty were performed in our hospital from July 2017 to December 2020, of which general anesthesia was about 5\% (24/458), intraspinal anesthesia was about 76\% (349/458), and ultrasound-guided nerve block was about $19 \%$ (85/458). About $47 \%$ of patients with ultrasound-guided nerve block were treated with anterior lumbar plexus block $(40 / 85)$. Forty patients with anterior lumbar plexus block were randomly divided into group A $(n=20)$ and group $B(n=20)$. Group A $(n=20)$ received ultrasound-guided anterior lumbar plexus combined with parasacral sciatic nerve block anesthesia. There were 8 males and 12 females, aged 65-83 years and weighing 45-83 kg. Twenty patients in group B were treated with anterior lumbar plexus block (10 males and 10 females, aged 67-85 years, weight $42-81 \mathrm{~kg}$ ). There was no significant difference in general data between the two groups $(\mathrm{P}>0.05)$. Inclusion and exclusion criteria: inclusion criteria: (1) patients and their families were aware of the study and voluntarily participated in the study; (2) The patient is conscious and cooperates well.; (3) in accordance with the American Association of Anesthesiologists (ASA) grade I III grade. Exclusion criteria: (1) mental abnormality and cognitive dysfunction, Elderly patients who are unable to cooperate or have difficulty in communication; (2) mental illness, peripheral nerve injury, liver and kidney insufficiency, coagulation dysfunction, malignant tumor and other diseases; (3) contraindications to regional block anesthesia, history of drug allergy, etc. All patients and their families signed an informed consent form and submitted it to the hospital ethics committee for examination and approval. The flow chart of the research object is shown in figure 1. 


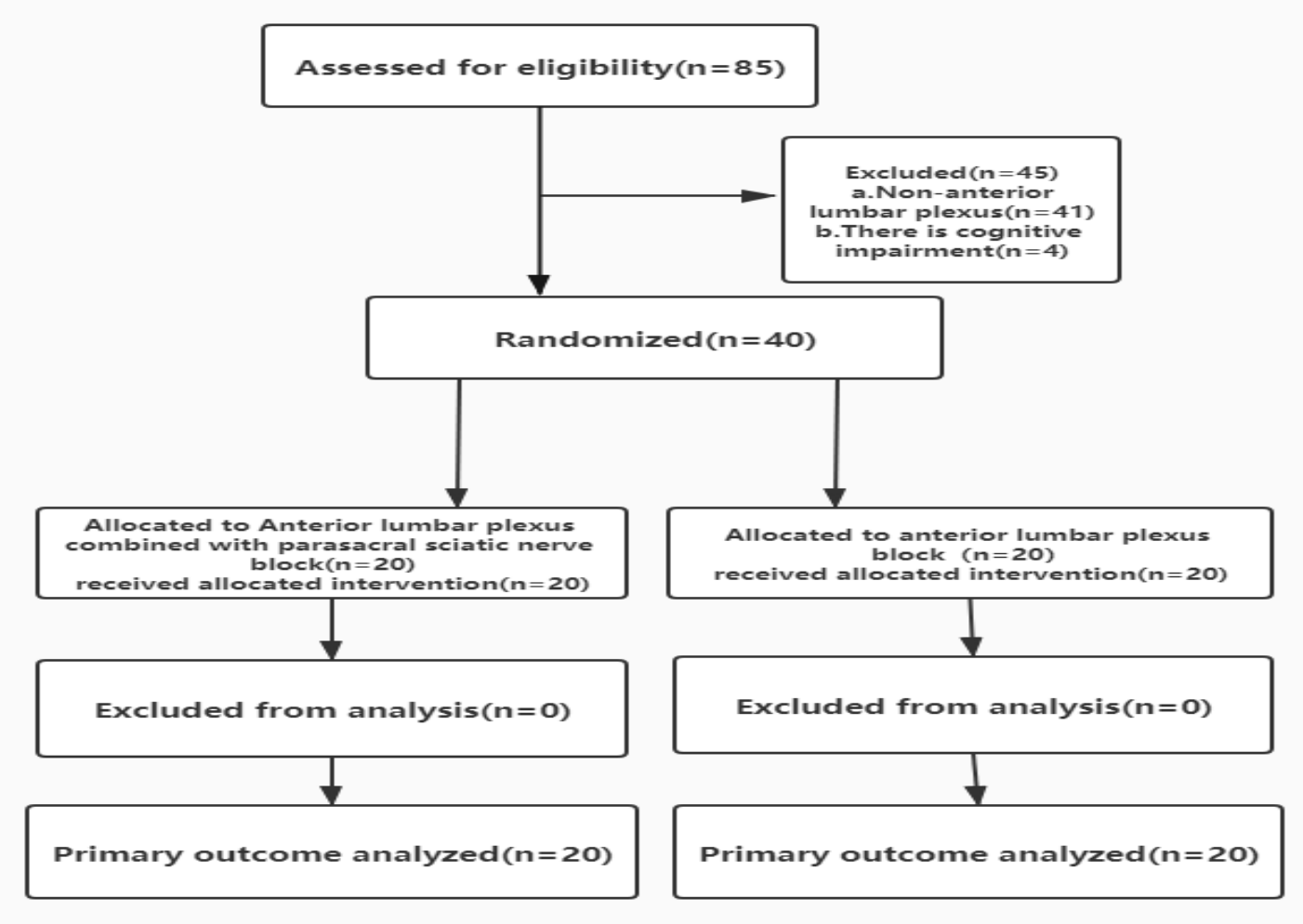

Figure 1. Research flow chart

\section{2 methods}

group A received ultrasound-guided anterior approach lumbar plexus combined with parasacral sciatic nerve block anesthesia, group B received ultrasound-guided anterior approach lumbar plexus block. Ultrasound equipment: GE portable color Doppler ultrasound system, puncture equipment: nerve stimulator plus nerve stimulation needle (Pajunk21G,100mm, Germany). After the nerve stimulator was successfully punctured at the initial current of $1 \mathrm{~mA}$, after the corresponding muscle contraction was induced by the nerve stimulator, and after adjusting the current to $0.3-0.5 \mathrm{~mA}$, to recover no blood, the local anesthetic could be injected. If the patient has local anesthetic poisoning during the injection of local anesthetic or after the completion of nerve block, it shall be treated according to the treatment procedure of local anesthetic poisoning, And changed to general anesthesia to complete the operation, such patients are not included in the study. Methods: anterior lumbar plexus block included femoral nerve, lateral femoral cutaneous nerve and obturator nerve. (1)Femoral nerve block: on the basis of keeping parallel to the inguinal ligament, the ultrasonic probe is placed in the groin, passing through the external side of the femoral artery, the cross section of the femoral nerve (fusiform, slightly hyperechoic) is visible, and the needle is inserted into the nerve plexus until the needle tip reaches the deep part of the femoral nerve. If it is determined that there is no blood, $0.5 \%$ ropivacaine $5 \mathrm{ml}$ can be injected, retreat the needle tip to the upper surface of the nerve, and then inject $0.5 \%$ ropivacaine $5 \mathrm{ml}$.(2)Lateral femoral cutaneous nerve block: the patient was lying on his back, routinely disinfecting the towel, placing the ultrasonic probe horizontally on the lower edge of the affected anterior superior iliac spine, looking for the lateral femoral cutaneous nerve in the medial side of the sartorius muscle and the superior 
edge of the iliac muscle, using a nerve plexus puncture needle $(21 \mathrm{G}, 100 \mathrm{~mm})$ to enter the needle from the outside, and the tip of the needle to reach the surface of the nerve. after determining that there was no blood, the local anesthetic $5 \mathrm{ml}$ was injected $0.5 \%$ ropivacaine. (3) Obturator nerve block: the ultrasonic probe was placed in the obturator and passed under the pubic muscle to see the cross section of the obturator nerve. The probe was moved to the foot side, about $1.5 \mathrm{~cm}$, and a screenshot of the obturator nerve in the middle part of the adductor brevis (anterior and posterior branches can be seen). Insert the nerve plexus puncture needle, make the tip of the needle reach the anterior and posterior branch of the obturator nerve, make sure that there is no blood, and inject $0.5 \%$ ropivacaine of $5 \mathrm{ml}$ respectively $[4]$. (4) Parasacral sciatic nerve block: at the puncture point, the posterior superior iliac spine and the greater trochanter of the femur were taken from the midpoint of the line to the downward $3 \mathrm{~cm}$. The frequency of the ultrasonic probe is $3.5-5.0 \mathrm{MHz}$. The location of the nerve plexus is determined by ultrasonic scanning, and the needle tip is ensured to be in the muscle space where the nerve runs. After the puncture is successful and no blood is drawn back, $0.5 \%$ ropivacaine $1 \mathrm{ml}$ is injected to observe the condition of the patient. If there is no adverse reaction, continue administration. During administration, the diffusion of local anaesthesia was observed under ultrasound. According to the diffusion, the position of the needle tip was adjusted to ensure that the nerve was effectively surrounded by the solution. The total dose of parasacral sciatic nerve block was $15 \mathrm{~mL}$.

\section{3 observation indicators}

(1) The block effects of patients in group An and B were observed, including the onset time of sensory block and motor block.(2) The heart rate and blood pressure at admission (T0), skin incision (T1), half hour after skin incision (T2) and skin suture

(T3) were observed and compared between the two groups. (3)The intraoperative dosage of dexmedetomidine and sufentanil in the two groups were recorded.(4) Observation after operation $1 \mathrm{~h} 、 4 \mathrm{~h} 、 8 \mathrm{~h} 、 16 \mathrm{~h} 、 24 \mathrm{hVAS}$ score and the time of getting out of bed for the first time.

\subsection{Statistical analysis}

SPSS 20 statistical software was used to analyze the data, and the measurement data were expressed by mean \pm standard deviation $(\overline{\mathrm{x}} \pm \mathrm{s})$. T-test was used for comparison between groups, ANOVA of repeated measurement data was used for comparison within groups, and $\mathrm{X}^{2}$ test was used for comparison of counting data. The difference was statistically significant $(\mathrm{P}<0.05)$.

\section{Results}

2.1 comparison of general data: there was no significant difference in sex ratio, age, BMI and ASA grade between the two groups $(\mathrm{P}>0.05$, Table 1$)$.

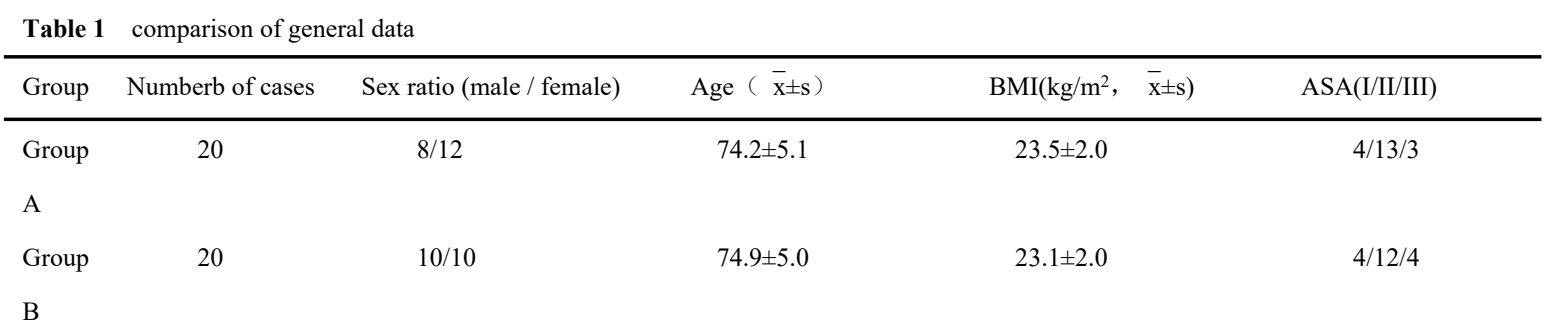




$\begin{array}{lll}\text { T value } & 0.501 & 0.672 \\ \text { P value } & 0.620 & 0.506\end{array}$

Note: group A: ultrasound-guided anterior lumbar plexus combined with parasacral sciatic nerve block group; group B: ultrasound-guided anterior lumbar plexus block group.

2.2 comparison of the onset time of sensory and motor block: the onset time of sensory block and motor block after ultrasound-guided nerve block in group A was significantly shorter than that in group B $(\mathrm{p}<0.05$, Table 2$)$.

Table 2 comparison of onset time of sensory and motor block

\begin{tabular}{|c|c|c|c|c|c|}
\hline Group & Number of cases & Onset time of sensory block & $(\min , \quad \bar{x} \pm s)$ & Onset time of motor block(min, & $\overline{\mathrm{x}} \pm \mathrm{s})$ \\
\hline Group A & 20 & $4.8 \pm 1.0$ & & $7.3 \pm 0.8$ & \\
\hline T value & & 39.736 & & 66.152 & \\
\hline$P$ value & & 0.000 & & 0.000 & \\
\hline
\end{tabular}

Note: group A: ultrasound-guided anterior lumbar plexus combined with parasacral sciatic nerve block group; group B: ultrasound-guided anterior lumbar plexus block group.

2.3 comparison of hemodynamic changes at different time points: there was no significant difference in heart rate and MAP between the two groups at $\mathrm{T} 0(\mathrm{P}>$ 0.05).The heart rate and MAP at T 1, T 2 and T 3 in group A were significantly lower than those in group $\mathrm{B}(\mathrm{P}<0.05$, Table 3$)$.

Table 3 comparison of hemodynamic changes at different time points $(\overline{\mathrm{x}} \pm \mathrm{s})$

\begin{tabular}{llllll}
\hline \multicolumn{1}{c}{ Index } & Group & Number of cases & T0 & T2 & T3 \\
\hline Heart rate (times / & Group A & 20 & $75.9 \pm 2.4$ & $77.7 \pm 4.3$ & $78.6 \pm 5.5$ \\
min) & & & & $91.3 \pm 5.4$ \\
& Group B & 20 & $77.1 \pm 2.8$ & $99.5 \pm 5.7$ & 7.455 \\
& T value & & 1.335 & 13.736 & 0.000 \\
& P value & & 0.190 & 0.000 & $88.9 \pm 3.9$ \\
MAP (mmHg) & Group A & 20 & $87.2 \pm 4.8$ & $92.0 \pm 3.9$ & $103.5 \pm 4.9$ \\
& Group B & 20 & $86.5 \pm 4.5$ & $107.4 \pm 4.9$ & 10.407 \\
& T value & & 0.475 & 10.985 & 0.000 \\
\hline
\end{tabular}

Note: group A: ultrasound-guided anterior lumbar plexus combined with parasacral sciatic nerve block group; group B: ultrasound-guided anterior lumbar plexus block group.T0: when entering the room, T1: when cutting the skin, T2: half an hour after the incision, T3: when sewing.

2.4 comparison of the dosage of sufentanil during operation and the time of getting out of bed for the first time after operation between the two groups: the dosage of sufentanil in group A was significantly less than that in group B $(p<0.05)$. At the same time, the first time of getting out of bed in group A was shorter than that in group B $(\mathrm{p}<0.05$, Table 4$)$.

Table 4 comparison of the dosage of sufentanil during operation and the time of getting out of bed for the first time after operation between the two groups

\begin{tabular}{lccc}
\hline Group & Number of cases & Intraoperative dosage of sufentanil $(\mathrm{ug}, \overline{\mathrm{x}} \pm \mathrm{s})$ & The first time to get out of bed after operation $(\mathrm{h}, \mathrm{X} \pm \mathrm{s})$ \\
\hline Group A & 20 & $6.4 \pm 1.2$ & $20.2 \pm 1.8$ \\
Group B & 20 & $21.5 \pm 2.3$ & $22.1 \pm 1.7$
\end{tabular}




$\begin{array}{lll}\text { T value } & 26.373 & 3.555 \\ \text { P value } & 0.000 & 0.001\end{array}$

Note: group A: ultrasound-guided anterior lumbar plexus combined with parasacral sciatic nerve block group; group B:

ultrasound-guided anterior lumbar plexus block group.

2.5 comparison of VAS scores at each time point after operation between the two groups: the scores of $1 \mathrm{~h}, 4 \mathrm{~h}, 8 \mathrm{~h}$ and $16 \mathrm{hVAS}$ in group A were significantly lower than those in group B $(\mathrm{p}<0.05)$.However, there was no significant difference in postoperative 24hVAS score between the two groups ( $\mathrm{p}>0.05$, table 5).

Table 5 comparison of VAS scores at each time point after operation between the two groups $(\mathrm{x} \pm \mathrm{s})$

\begin{tabular}{|c|c|c|c|c|c|c|}
\hline Group & Number of cases & $\begin{array}{l}\text { 1h after } \\
\text { operation }\end{array}$ & $\begin{array}{l}\text { 4h after } \\
\text { operation }\end{array}$ & $\begin{array}{l}8 \mathrm{~h} \text { after } \\
\text { operation }\end{array}$ & $\begin{array}{l}16 \mathrm{~h} \quad \text { after } \\
\text { operation }\end{array}$ & $24 \mathrm{~h}$ after operation \\
\hline Group A & 20 & $1.9 \pm 0.5$ & $1.4 \pm 0.5$ & $2.3 \pm 0.5$ & $3.0 \pm 0.6$ & $2.7 \pm 0.6$ \\
\hline Group B & 20 & $2.3 \pm 0.7$ & $3.0 \pm 0.7$ & $4.0 \pm 0.6$ & $4.0 \pm 0.6$ & $2.5 \pm 0.5$ \\
\hline T value & & 3.616 & 8.370 & 10.187 & 5.867 & 1.109 \\
\hline$P$ value & & 0.001 & 0.000 & 0.000 & 0.000 & 0.274 \\
\hline
\end{tabular}

Note: group A: ultrasound-guided anterior lumbar plexus combined with parasacral sciatic nerve block group; group B: ultrasound-guided anterior lumbar plexus block group.

\section{Discussion}

Most of the patients undergoing hip arthroplasty are elderly patients with poor cardiopulmonary function and commonly used intraspinal anesthesia, but there are more postoperative complications [5]. Patients need a long time to recover and reduce the curative effect of the operation. at the same time, the use of routine anticoagulants also increased the risk of intraspinal anesthesia. General anesthesia does not block the pain afferent to the operation area, and nociceptive stimuli such as surgical stress can still be transmitted to the center to cause hemodynamic fluctuations, and general anesthesia has a great impact on cardiopulmonary function, resulting in postoperative respiratory insufficiency, secondary pulmonary infection and so on. Compared with general anesthesia and intraspinal anesthesia, peripheral nerve block can temporarily lose the sensorimotor function in the innervated area and block the upload of nociceptive stimuli, which has little effect on the whole body and stable hemodynamics. At the same time, it has the advantages of perfect postoperative analgesia and so on. In addition, intraspinal anesthesia is often used in clinic to assist patients in operation, but in many elderly patients, intraspinal anesthesia is limited due to severe bending of the spine or hyperosteogeny [6]. it is easy to cause the failure of intraspinal anesthesia ${ }^{7}$.Therefore, how to choose an effective way of anesthesia has always been the focus of clinical attention. With the development of precision medicine, anaesthesia technology must also develop to precision anesthesia. Accurate anesthesia is beneficial to the prognosis of patients, reduce intraoperative and postoperative anesthesia complications, reduce patients' pain, facilitate patients' early recovery, and nerve block can provide accurate anesthesia $[8]$. At present, ultrasound-guided three-in-one femoral nerve block (FNB) and iliac fascial space block (FICB), are commonly used for anesthesia and analgesia in hip arthroplasty [9, 10 ].Because of the complexity of hip innervation, it is difficult to use nerve block 
anesthesia in hip arthroplasty. Four nerves: obturator nerve, femoral nerve, sciatic nerve and lateral femoral cutaneous nerve innervate the nerve supply of the hip joint [11 ] . It is reported that $81 \%$ success rate of femoral nerve block and $96 \%$ success rate of lateral femoral cutaneous nerve block can be obtained by three-in-one block. In iliofascial block, the obturator nerve block rate is only $28 \%$, and the obturator nerve is an important hip innervation branch, incomplete block can lead to intraoperative pain, stress and other nociceptive reactions [12].The main areas of lumbar plexus block are femoral nerve, lateral femoral cutaneous nerve, obturator nerve, ilioabdominal nerve and ilioinguinal nerve.Anatomically, the space of the psoas major muscle is large, and the distribution of these nerves in the psoas major muscle space is not concentrated. The ilioabdominal and ilioinguinal nerves are located at the top of the lumbar plexus and separate from the lumbar plexus. there is also a certain distance between the lateral femoral cutaneous nerve and the femoral nerve and obturator nerve at the level of the transverse process of the third or fourth lumbar vertebrae commonly punctured in the lumbar plexus.Even if the posterior lumbar plexus is injected accurately, it still needs a large dose of local anesthetic to block it. And the depth of posterior lumbar plexus puncture is difficult, and it is difficult for young doctors to master. Posterior lumbar plexus block has the risk of psoas major muscle hematoma, epidural diffusion and bilateral block $[13,14]$. Ultrasound-stimulator double-guided multi-point anterior lumbar plexus block can reduce the amount of auxiliary inhalation or intravenous drugs, without the risk of psoas major muscle hematoma, epidural diffusion and bilateral block, and is not affected by the use of anticoagulants. It is safer and more effective for elderly patients [15].In this study, patients in both groups were supplemented with dexmetomidine and sufentanil during operation, and the doses of the two drugs were adjusted according to the specific conditions of the patients. the heart rate and MAP in group A were significantly lower than those in group B at T1, T2 and T3, indicating that group A could significantly reduce the pain caused by incision traction during skin incision or during operation, thus filling the incomplete block caused by anterior lumbar plexus block alone. The dosage of sufentanil in group A was significantly less than that in group B, because the pain block of incision after parasacral sciatic nerve block was more perfect, so the dosage of opioids was reduced, so the recovery time after operation was significantly shortened. The VAS score of group A was lower than that of group B at $1 \mathrm{~h}, 4 \mathrm{~h}, 8 \mathrm{~h}$ and $16 \mathrm{~h}$ after operation, suggesting that the effect of postoperative analgesia in group A was better, and there was no significant difference in VAS score between the two groups 24hours after operation, indicating that the effect of nerve block disappeared 24hours after operation. The first time of getting out of bed in group An is earlier than that in group B, indicating that the effect of early postoperative analgesia is perfect, which is beneficial to patients' early functional exercise and promote the recovery of joint function, and early postoperative activity can reduce the incidence of deep venous thrombosis in elderly patients. Ultrasound-guided nerve block only anesthetized one side of the lower limb, compared with intraspinal anesthesia, the level of anesthesia was too high, the range was too wide, the inhibition of sympathetic nerve was too strong, and the hyperactivity of vagus nerve caused nausea and 
vomiting. There is no high anesthesia level of intraspinal anesthesia leading to postoperative urinary retention. In addition, under the action of ultrasonic technology, it can ensure the accuracy of the location of nerve block and intuitively understand the diffusion law of local anesthetics in the specific conditions of the puncture site (blood vessels, muscles, nerves, etc.), so as to control the dosage of local anesthetics and protect blood vessels and nerves. reduce the risk of adverse reactions [ 16 ] .Ultrasound-guided nerve block is safe and effective to control early postoperative pain [17] and reduces patients' fear after hip arthroplasty. In addition, we found that inguinal iliac fascia block may lead to longer sensory block. We speculate that the prolongation of this block time is due to the absence of blood vessels in the iliofascial septum $[18]$. Most patients often experience moderate to severe postoperative pain after hip arthroplasty. Postoperative pain control has a significant impact on patients going to the ground earlier, starting physiotherapy and better functional recovery. In addition, effective pain control will shorten the length of stay in hospital and the risk of thrombotic events, and improve patient satisfaction [ 19 ] .To sum up, ultrasound-guided anterior lumbar plexus combined with parasacral nerve block combined with superficial sedation in elderly patients with hip arthroplasty can better maintain the stability of circulation in elderly patients, reduce the amount of opioids during operation, and wake up quickly after operation. the effect of early postoperative analgesia is more perfect and can play a role in rapid rehabilitation. It is a safe anesthetic scheme which can improve the comfort of patients.

\section{Conclusion}

Although anterior lumbar plexus combined with parasacral sciatic nerve block and simple anterior lumbar plexus block can provide good pain treatment for patients with hip replacement, anterior lumbar plexus combined with parasacral sciatic nerve block has faster effect and better analgesia.

\section{Acknowledgements}

We would like to thank all respondents for their generous participation.

\section{Availability of data and materials}

The data will not be made available in order to protect the participants' identity.

\section{Author Contributions}

Wei Wu, Hai-bin Huang, Ying Mao is involved in collecting data, Yong-xing Tan is involved in data analysis, and $\mathrm{Wei} \mathrm{Wu}, \mathrm{Wen}-\mathrm{hao} \mathrm{Bu}$ is involved in drafting or revising articles. All the authors agreed to the journal that submitted the paper, finally approved the version to be published, and agreed to be responsible for all aspects of the work.

\section{Funding}

None

\section{Consent for publication}

Signed informed consent was obtained from each patient.

\section{Competing interests}

The authors indicated no potential conflicts of interest. 


\section{Ethical approval and informed consent}

this experiment was examined and approved by the Ethics Committee of CR \& WISCO General Hospital. Prior to the recruitment of the study, obtain written and informed consent from all participants. This experiment was conducted according to the Helsinki Declaration and approved by the Ethics Committee of CR \& WISCO General Hospital(Review approval No.: HRWGZYY20210010).

\section{Clinical trial registration numbers and date of registration}

We have registered with the WHO International Clinical Trial Registry Platform. Date of registration and Clinical trial registration numbers:2021/02/16; ChiCTR2100043422.

\section{Availability of data and materials}

The datasets generated during and analysed during the current study are not publicly available in order to protect the participants' identity but are available from the corresponding author on reasonable request.

\section{References}

1.Zhou J. Effect of ultrasound-guided lumbar plexus combined with parasacral sciatic nerve block anesthesia in internal fixation of femoral neck fracture in elderly patients. Series of Contemporary Medicine.2020, 18 (19): 35-19.

2.Luo FY, Yu XJ, Fan YL, et al. Clinical application of ultrasound-guided anterior lumbar plexus combined with superior gluteal nerve block in hip arthroplasty in the elderly. International Journal of Anesthesiology and Resuscitation.2019 Journal 40 (7): 637-613.

3.Højer Karlsen AP, Geisler A, Petersen PL, Mathiesen O, Dahl JB. Postoperative pain treatment after total hip arthroplasty: a systematic review.Pain. 2015 Jan;156(1):8-30.

4.Qin S, Yang KF, Liao JM. Observation on the anesthetic effect of ultrasound-guided anterior lumbar plexus combined with sciatic nerve block in lower limb surgery. Jilin Medicine, 2019. 40 (8): 1755-1757.

5.Ding EL, He T. Anesthetic effect of ultrasound-guided anterior lumbar plexus block combined with sciatic nerve block in lower limb surgery. Shanxi Medical Journal, 2020, 49 (12): 1554-1556.

6.Yao WL. Application value of ultrasound-guided lumbar plexus combined with parasacral sciatic nerve block in internal fixation of femoral neck fracture in the elderly. Frontier of Medicine.2019 (27): 139-140.

7.Sun XF, Pang B. Effect of ultrasound-guided lumbar plexus combined with parasacral sciatic nerve block in internal fixation of femoral neck fracture in elderly patients. China Medical Innovation.2020 and 17 (4): 47-50.

8.Wang CG. To investigate the effect of ultrasound-guided lumbar plexus-sciatic nerve block in unilateral lower limb surgery in elderly patients. Chinese and Foreign Medical Research.2017 15 (3): 28-30. 
9.Barrington JW, Halaszynski TM, SinatraRS. Expert Working Group On Anesthesia, Orthopaedics Critical Issues In Hip and Knee Replacement Arthroplasty FTPerioperative pain management in hip and kneereplacement surgery. Am J Orthop (Belle Mead, NJ) 2014;43(4 suppl):S1-16.

10.Topal FE, Bilgin S, Yamanoglu A, Karakaya Z, Payza U, Akyol PY, Aslan C, Aksun M. The Feasibility of the Ultrasound-Guided Femoral Nerve Block Procedure with Low-Dose Local Anesthetic in Intracapsular and Extracapsular Hip Fractures. J Emerg Med. 2020 Apr;58(4):553-561.

11.Yu B, He M, Cai GY, Zou TX, Zhang N. Ultrasound-guided continuous femoral nerve block vs continuous fascia iliaca compartment block for hip replacement in the elderly: A randomized controlled clinical trial (CONSORT). Medicine (Baltimore). 2016 Oct;95(42):e5056.

12.Zhang DZ, Chang Y, Yang QG, et al. Comparison of ultrasound-guided multi-point block and single-point block in iliofascial space at different angles. Journal of Clinical Anesthesiology. 2015,31 (1): 50-52.

13.Luo Y, Cao Q. Comparative observation on the effect of lumbar plexus sciatic nerve block guided by ultrasound combined with nerve stimulator and combined spinal-epidural anesthesia in the operation of femoral intertrochanteric fracture in the elderly. Electronic Journal of Clinical Medical Literature.2017,4(49): 9520-9521.

14.Zuo D, Zhou M. Application of ultrasound-guided lumbar plexus combined with parasacral sciatic nerve block in internal fixation of femoral neck fracture in the elderly. Journal of Trauma surgery. 2017,19 (7): 503-507.

15.Feng BY, Zhang XL, you YY, et al. Application of multi-point anterior lumbar plexus block under the dual guidance of ultrasound and stimulator in the operation of proximal femoral fracture in the elderly. Chinese practical Medicine.2018 13 (5): $8-10$

16.Yu JY, Wang C, Shao L, et al. Effect of ultrasound-guided lumbar plexus combined with sciatic nerve block on postoperative infection and immune function in elderly patients with hip fracture. Chinese Journal of Hospital Epidemiology. 2018 focus 28 (2): 229.

17.Cappelleri G, Ambrosoli AL, Gemma M, Cedrati VLE, Bizzarri F, Danelli GF. Intraneural Ultrasound-guided Sciatic Nerve Block: Minimum Effective Volume and Electrophysiologic Effects. Anesthesiology. 2018 Aug;129(2):241-248.

18.Bravo D, Layera S, Aliste J, Jara Á, Fernández D, Barrientos C, Wulf R, Muñoz G, Finlayson RJ, Tran Q. Lumbar plexus block versus suprainguinal fascia iliaca block for total hip arthroplasty: A single-blinded, randomized trial. J Clin Anesth. 2020 Nov;66:109907.

19.Wang X, Sun Y, Wang L, Hao X. Femoral nerve block versus fascia iliaca block 
for pain control in total knee and hip arthroplasty: A meta-analysis from randomized controlled trials. Medicine (Baltimore). 2017 Jul;96(27):e7382. 


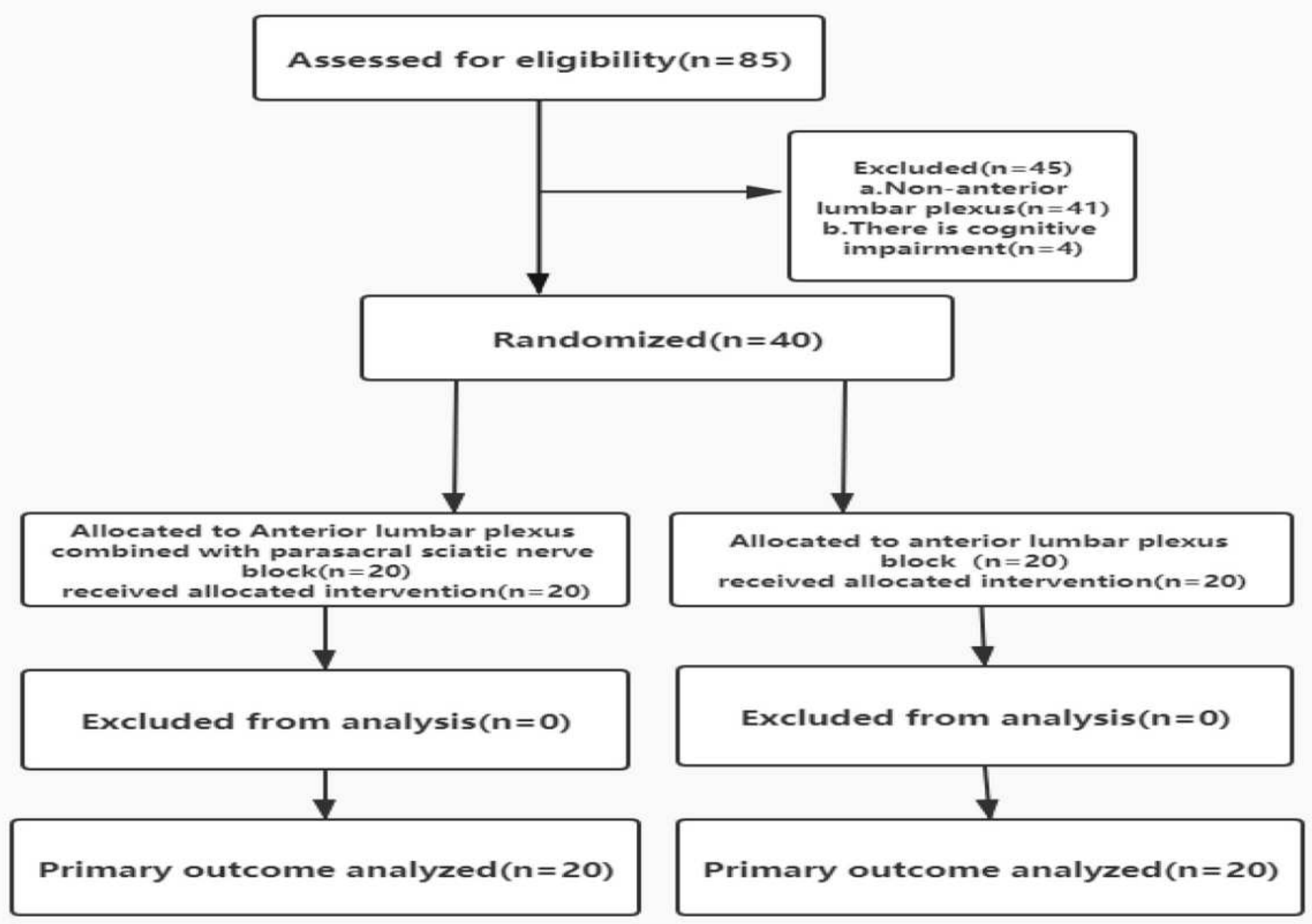

Figure 1

Research flow chart 